\title{
Avaliação da efetividade do tratamento termoquímico de carbonitretação em engrenagens de motocicletas
}

\author{
Effectiveness evaluation of thermochemical \\ treatment of carbonitriding in motorcycle gears
}

\author{
Hektor Oliveira Borges ${ }^{1}$, Angelo Ores Bonamigo ${ }^{1}$, Raí Lima Vieira ${ }^{1}$, \\ Jorge Luis Braz Medeiros ${ }^{1}$, Luciano Volcanoglo Biehl ${ }^{1}$, José de Souza ${ }^{2}$
}

\footnotetext{
${ }^{1}$ Universidade Federal do Rio Grande - FURG, Programa de Pós-Graduação em Engenharia Mecânica, Av. Itália, Km 8, s/n, CEP: 96203-900, Rio Grande, Rio Grande do Sul, Brasil.

${ }^{2}$ Fundação Escola Técnica Liberato Salzano Vieira da Cunha (FETLSVC), Diretoria de Pesquisa e Produção Industrial (DPPI), Rua Inconfidentes, 395, CEP: 93340-140, Novo Hamburgo, Rio Grande do Sul, Brasil.

e-mail: hektor.borges@furg.br, angelo.bonamigo@gmail.com,rai@furg.br, jorge.braz@ furg.br, lucianobiehl@furg.br, josesouza@liberato.com.br
}

\section{RESUMO}

O objetivo desse estudo é avaliar a efetividade do tratamento termoquímico de carbonitretação empregado em engrenagens de motocicletas, para possível substituição de um aço médio carbono, por um aço de baixo carbono. Para isto foi determinada à composição química do material, analisado a microestrutura, verificado o perfil de microdureza e observando-se a conformidade dos ensaios. As engrenagens utilizadas no tratamento termoquímico foram produzidas com aço SAE 1015, as quais foram submetidas à austenitização na temperatura de $880^{\circ} \mathrm{C}$ durante 6 horas, utilizando atmosfera controlada com potencial de carbono de $1 \%$ e reduzido volume de $\mathrm{NH}_{3}$, sendo posteriormente temperada em óleo e revenidas a uma temperatura de $180{ }^{\circ} \mathrm{C}$ durante um período de 1 hora. A microestrutura do núcleo apresentou ferrita, perlita e martensita de baixo carbono junto aos contornos de grão. Já na camada carbonitretada detectou-se nitretos de elevada dureza e martensita de alto carbono associada a pequeno volume de austenita retida. Através da técnica de difração de raios-X, confirmou-se a presença de austenita retida na superfície das amostras. A microdureza máxima atingida foi de $949 \mathrm{HV}_{0,1}$ e a profundidade de camada carbonitretada de $0,8 \mathrm{~mm}$. Conclui-se que a utilização de um aço baixo carbono e não ligado pode-se tornar uma rota de fabricação para engrenagens, quando submetidos ao tratamento termoquímico de carbonitretação. A presença de potencial de carbono muito elevado é elemento favorável para formação de austenita retida.

Palavras-chave: DRX, perfil de microdureza, tratamento termoquímico

\section{ABSTRACT}

The objective of this study is to evaluate the effectiveness of the thermochemical treatment of carbonitriding employed in motorcycle gears for the replacement of a medium carbon steel to a low carbon steel. For this, the chemical composition of the material was determined, the microstructure analyzed, traced the microhardness profile and verified the conformity of the tests. As the gears used in the thermochemical treatment were produced with SAE 1015 steel, such as those that were subjected to austenitization at a temperature of $880^{\circ}$ $\mathrm{C}$ for 6 hours, using a controlled atmosphere with $1 \%$ carbon potential and low $\mathrm{NH}_{3}$ volume, cooled in oil and tempered at $180^{\circ}$ for 1 hour. A presented microstructure of the ferrite and perlite core and a high hardness nitrided carbonitride layer and high carbon martensite associated with the austenite retention volume. The x-ray diffraction technique confirmed the presence of austenite retained on the sample surface. A maximum depth reached was $949 \mathrm{HV}_{0.1}$ and a carbonitride layer depth of $0.8 \mathrm{~mm}$. Thereby, the use of low carbon and unalloyed steel could be a manufacturing route for gears when using carbonitride thermochemical treatment. The presence of very high carbon potential is favorable for austenite retina formation.

Keywords: XRD, microhardness profile, thermochemical treatment 


\section{INTRODUÇÃO}

Os tratamentos termoquímicos são utilizados para melhorar as propriedades superficiais dos materiais com o aumento da dureza, da resistência mecânica, da resistência ao desgaste e também da resistência à fadiga. $\mathrm{O}$ objetivo desses tratamentos é a difusão elementos químicos intersticiais, como carbono e nitrogênio, na superfície dos aços [1,2].

De uma forma geral, nos tratamentos termoquímicos de cementação e carbonitretação, existe a utilização de aços de baixo carbono, porém com maior temperabilidade. Estes aços são classificados na classe baixo carbono e baixa liga como o SAE 8620 ou SAE 4320, que apresentam maior temperabilidade de núcleo pela adição de elementos de liga.

Estes aços, com maior temperabilidade a variação de resistência mecânica, apresentam uma transição mais suave entre a superfície e o núcleo, porém com menor tenacidade do conjugado.

Já os aços com baixa temperabilidade possuem elevada tenacidade de núcleo, minimizando o efeito de fragilização de regiões com menores espessuras e com elevadas profundidades de camada de difusão.

Em busca do desenvolvimento de ciclo alternativo de fabricação, estudou-se a substituição do aço SAE 1045 temperado e revenido pelo aço SAE 1015 submetido a um tratamento de carbonitretação, de modo que o desempenho dessas engrenagens seja aumentado, possibilitando a elevação da resistência mecânica e ao desgaste superficial.

Existem diferentes tipos de tratamentos termoquímicos, que são amplamente utilizados pela indústria automotiva, sendo que cada um apresenta as suas vantagens e desvantagens. Os processos mais empregados são os de cementação, carbonitretação e nitretação. Estes podem ser realizados em diferentes atmosferas como líquida, gasosa e plasma [2,3].

Os tratamentos termoquímicos são processos cuja à finalidade é obter um endurecimento na superfície dos aços e ao mesmo tempo em que o núcleo permanece tenaz. A combinação de uma superfície endurecida com um núcleo dúctil é favorável para peças como engrenagens, pois o aumento da dureza superficial reduz o desgaste e com o núcleo dúctil é capaz de absorver os esforços durante operações cíclicas. Os tratamentos termoquímicos também são utilizados para aumentar a resistência à corrosão, à fadiga, devido ás tensões compressivas e à oxidação em elevadas temperaturas [4-6].

No processo de carbonitretação gasosa existe o endurecimento da superfície do aço através da difusão de carbono e nitrogênio. $\mathrm{O}$ carbono é disponibilizado a partir de atmosfera sintética a base de propano e metanol, sendo nitrogênio gerado a partir dissociação da amônia $\left(\mathrm{NH}_{3}\right)$, de forma que o nitrogênio difunde no aço simultaneamente com o carbono. O tempo e a temperatura desse processo são geralmente menores, quando comparadas com a cementação gasosa e superiores a nitretação gasosa. Usualmente esse processo é seguido de um tratamento térmico de tempera e revenimento [5, 7].

Na carbonitretação as deformações são menores na etapa de têmpera, comparando-se a cementação, devido às temperaturas de austenitização serem mais baixas. A taxa de fluxo de amônia utilizada no forno também influência na profundidade da camada e a formação de precipitados [7, 8].

\section{MATERIAIS E MÉTODOS}

Para determinar a composição química das amostras, foi realizada espectrometria de emissão óptica em um equipamento modelo Foundry-Master Pro, da Oxford Instruments

O processo de carbonitretação consistiu em austenitizar na temperatura até $880{ }^{\circ} \mathrm{C}$ em atmosfera controlada com potencial de carbono de $1 \%$ e amônia $\left(\mathrm{NH}_{3}\right)$ dissociada durante um período de 6 horas. O resfriamento na têmpera foi realizado em óleo acelerado com severidade de $110^{\circ} \mathrm{C} / \mathrm{s}$, sendo realizado posteriormente o revenimento a uma temperatura de $180{ }^{\circ} \mathrm{C}$, pelo período de 1 hora, para redução das tensões na camada carbonitretada.

As amostras foram posteriormente cortadas e embutidas para o manuseio, além de facilitar o processo de preparação que constituiu de lixamento, polimento e ataque seguindo a norma ASTM E3-11 (2001) [9]. O polimento foi feito com disco giratório de feltro e aplicado pasta de diamante como abrasivo. Para o ataque microestrutural foi utilizado o reagente Nital $2 \%$.

A análise macroestrutural foi realizada em microscópio Olympus GX 51S e o microscópio eletrônico de varredura (MEV) com microssonda EDS (Energy Dispersive Spectroscopy) da marca Jeol, modelo JSM6610LV, em que foi possível utilizar dois modos de obtenção de imagens, o Secondary Electron Induced (SEI) e o Backscatter Electron Composite (BEC). No modo SEI, através de emissão de elétrons secundários resultantes da interação do feixe eletrônico com o material da amostra, foi possível obter a imagem através do 
contraste gerado pelo relevo da amostra. Utilizando o modo BEC à imagem é gerada através de elétrons retro espalhados que fornecem diferentes informações em relação ao contraste, dessa forma, a geração da imagem é feita considerando o relevo da amostra e o número atômico dos elementos presentes [10].

Para a determinação do perfil de microdureza foi utilizado microdurômetro da marca Shimadzu, modelo HV2. O penetrador empregado é do tipo Vickers, aplicando uma carga de $0,1 \mathrm{~kg}$ durante um tempo de 10 segundos. O ensaio foi realizado de acordo com a norma ASTM E384-17 [11].

Utilizando difração de raios-x foi possível determinar a existência das fases nas amostras. Os padrões de difração foram captados com o $2 \theta$ variando de $30^{\circ}$ a $120^{\circ}$. O comprimento de onda da fonte geradora do equipamento é $1,5418 \AA$ [12].

\section{RESULTADOS}

\subsection{Composição Química}

Após realizar a espectrometria de emissão óptica verificou-se os elementos químicos presentes nas amostras, e confirmou-se que as amostras foram produzidas com o aço SAE 1015. A Tabela 1 apresenta a média dos resultados obtidos, após três ensaios. Destaca-se a presença de elementos residuais de sucata como $\mathrm{Cr}$, Mo e $\mathrm{V}$, que contribuem para o aumento da temperabilidade da camada carbonitretada e de núcleo [8].

Tabela 1: Composição química das amostras produzidas com o aço SAE 1015.

\begin{tabular}{c|c|c|c|c|c|c|c}
\hline Elemento & $\mathrm{C}$ & $\mathrm{Si}$ & $\mathrm{Mn}$ & $\mathrm{P}$ & $\mathrm{S}$ & $\mathrm{Cr}$ & $\mathrm{Mo}$ \\
\hline Média & 0,179 & 0,0277 & 0,541 & 0,0074 & 0,0125 & 0,013 & 0,0375 \\
\hline Elemento & $\mathrm{Al}$ & $\mathrm{Co}$ & $\mathrm{Cu}$ & $\mathrm{Nb}$ & $\mathrm{Ti}$ & $\mathrm{V}$ & $\mathrm{W}$ \\
\hline Média & 0,0601 & 0,0032 & 0,0073 & 0,0047 & 0,0024 & 0,001 & 0,0075 \\
\hline
\end{tabular}

\subsection{Análise Microscopia Óptica e Eletrônica de Varredura - EDS}

Após a aplicação da carbonitretação observa-se na Figura 1, da direita para esquerda, a presença de nitretos no início da camada (A), seguida uma camada de martensita de alto carbono, com nitretos precipitados (B) e por último uma camada com martensita de baixo carbono (C) similares ao encontrado por Taweejun et al. (2017) [5].

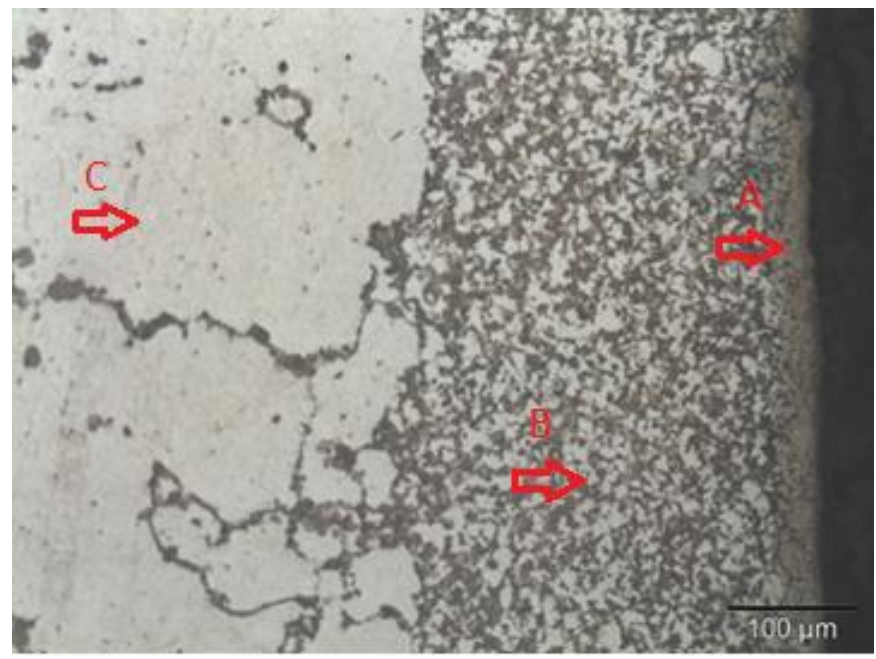

Figura 1: Comportamento da carbonitretada, temperada e revenida, identificando-se a distribuição morfológica.

Com o auxílio do MEV foi possível obter uma maior magnificação das amostras e analisar o núcleo temperado e revenido. Na figura 2, verifica-se na região mais escura constituída por ferrita e a mais clara martensita de baixo carbono. Esta microestrutura representa a morfologia típica de aços hipoeutetóides de baixo carbono em que essa condição é favorável à difusão de carbono na superfície, sendo o aço indicado 
para cementação ou carbonitretação $[5,13]$. A presença de ferrita e martensita de baixo carbono contribui para a elevada tenacidade de núcleo [8].

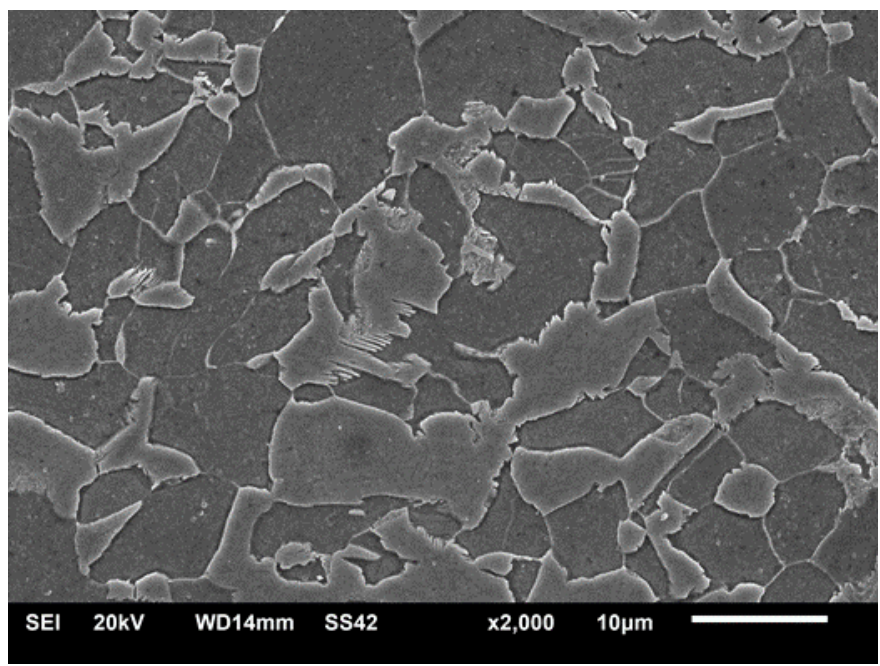

Figura 2: Núcleo da amostra de aço SAE 1015, após a aplicação do tratamento termoquímico.

Com a utilização de outro contraste, o Backscatter Electron Composite, foi possível visualizar de forma mais clara as microestruturas presentes na amostra. Na Figura 3 apresenta as microestruturas verificadas, sendo as regiões escuras a ferrita e as regiões claras a martensita de baixo carbono.

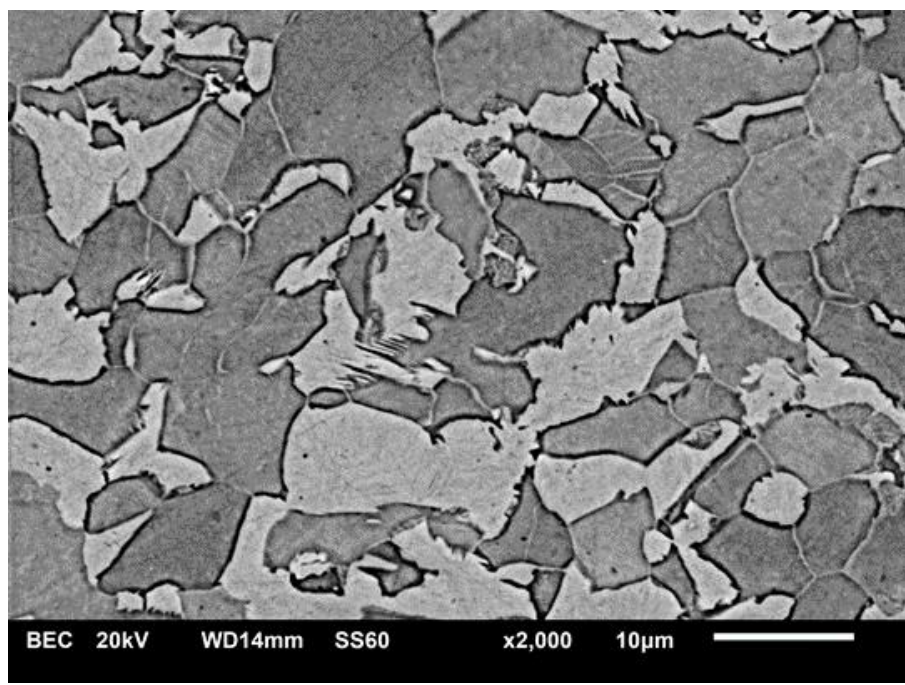

Figura 3: Núcleo da amostra base com contraste BEC constituída por martensita de baixo carbono e ferrita.

A zona de transição da camada carbonitretada apresenta a microestrutura de martensita de baixo carbono e ferrita, que pode ser melhor observada na Figura 4 [1]. 


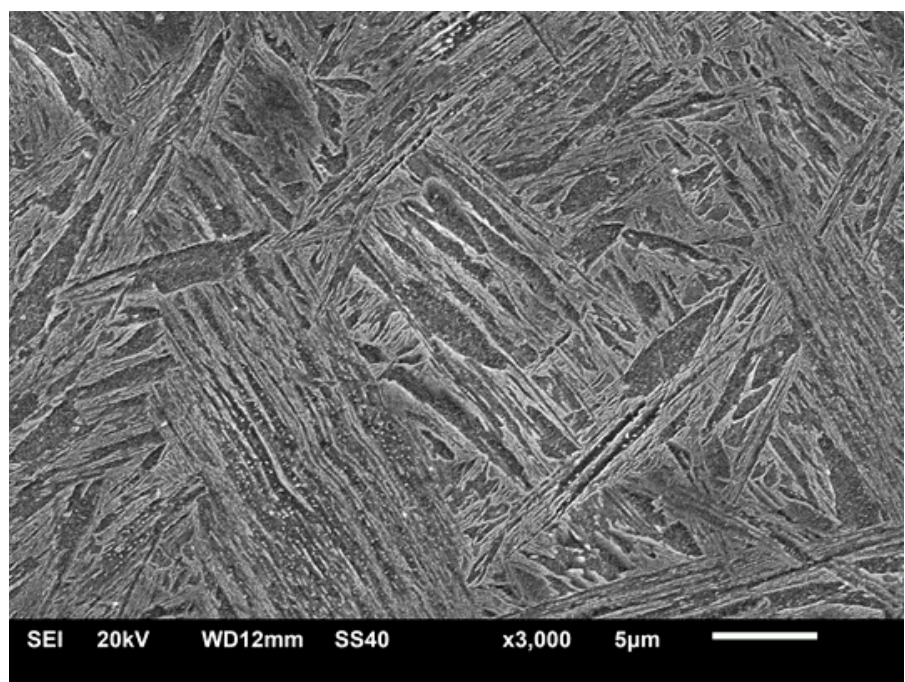

Figura 4: Microestrutura da zona de transição entre a camada carbonitretada e o núcleo.

Na Figura 5 que mostra a camada carbonitretada utilizando o modo SEI é possível observar a camada de nitretos presente na superfície da engrenagem, que na imagem aparece sendo a linha branca na borda inferior da figura. Podemos notar a formação da camada de nitretos e logo após a camada de martensita de alto carbono e nitretos $[1,13]$.

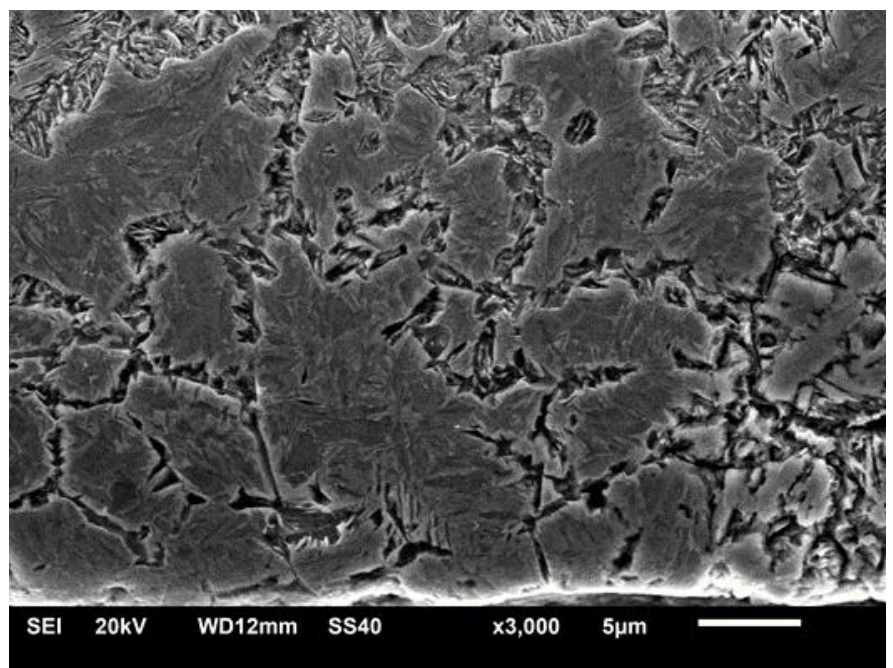

Figura 5: Camada Carbonitretada observando-se na borda superficial camada de nitretos, seguido de camada carbonitretada.

Através da microssonda EDS confirmou-se a presenta de carbono e nitrogênio na camada carbonitretada, consolidando a efetividade do tratamento. Na figura 6 determina o espectro encontrado na camada carbonitretada. 


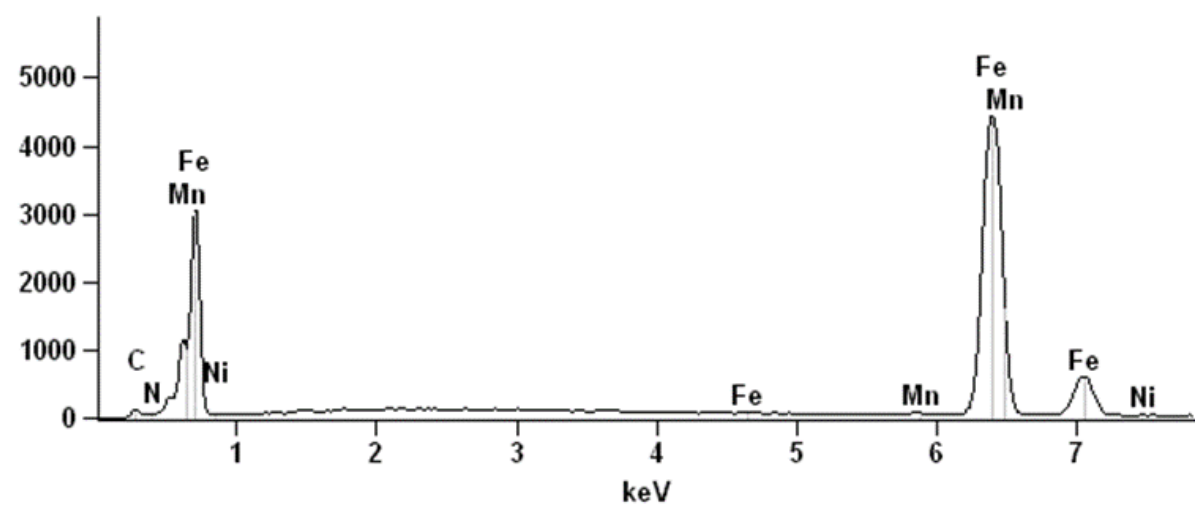

Figura 6: Elementos encontrados no ensaio qualitativo e semiquantitativo EDS, demostrando a presença de carbono e nitrogênio na camada carbonitretada.

\subsection{Análise Perfil de Microdureza}

O perfil de microdureza do aço carbonitretado é apresentado na Figura 7. O pico de microdureza foi constatado na profundidade $0,4 \mathrm{~mm}$ com valor $949 \mathrm{HV}_{0,1}$, reduzindo gradativamente a partir deste valor em direção ao núcleo. A microdureza detectada na camada mais superficial do material foi inferior ao do pico, pela presença de austenita retida. Em estudo semelhante, Kusmanov (2016) [14] obteve um perfil de microdureza similar ao encontrado.

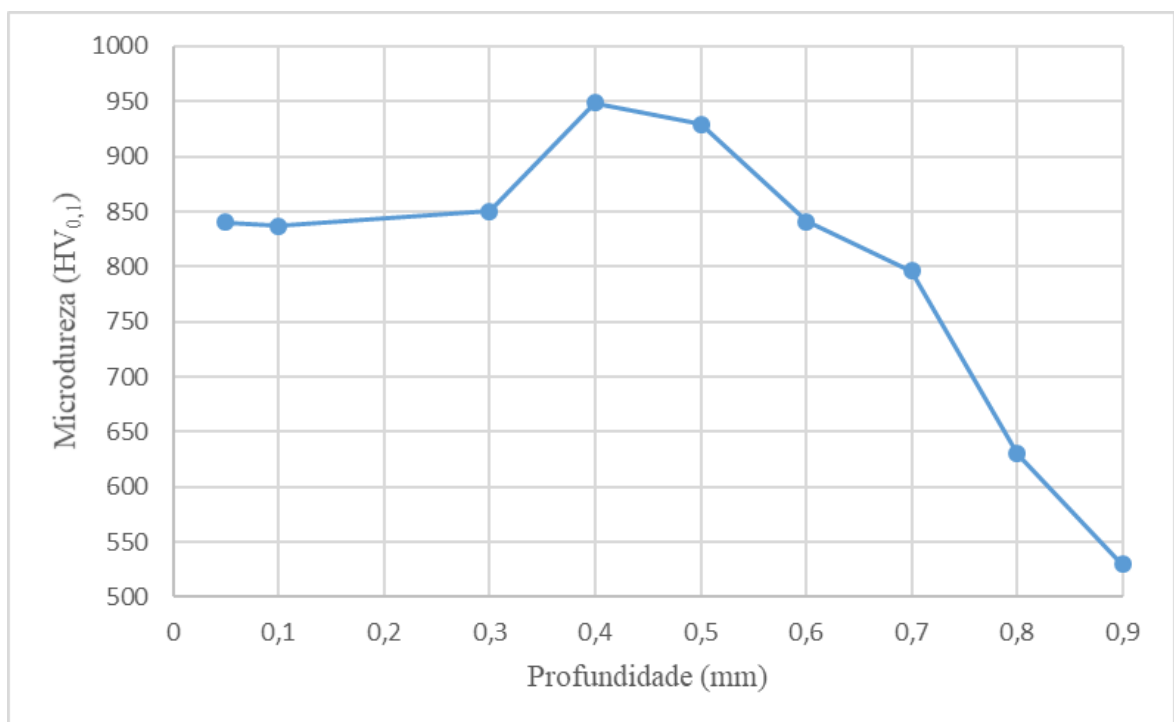

Figura 7: Perfil de microdureza $\left(\mathrm{HV}_{0,1}\right)$ da amostra carbonitretada verificando-se comportamento médio das amostras.

Segundo a norma DIN 50190, a profundidade da camada carbonitretada termina quando a microdureza atingir $550 \mathrm{HV}$, dessa forma a profundidade da camada alcançou 0,8 $\mathrm{mm}$ [13].

\subsection{Análise do Comportamento da zona de Transição e Camada Carbonitretada DRX}

Verificou-se no difratograma das amostras, na zona de transição, a presença de picos relacionados a presença de martensita e ferrita. A presença da austenita retida na camada carbonitretada também foi confirmada através do ensaio de difração de raios-X. A Figura 8 apresenta o difratograma encontrado para amostra, apresentando difração nos planos correspondentes com a ferrita na zona de transição (110) $\alpha,(200) \alpha,(211) \alpha,(220) \alpha$ e

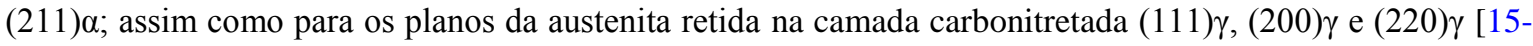
17]. 


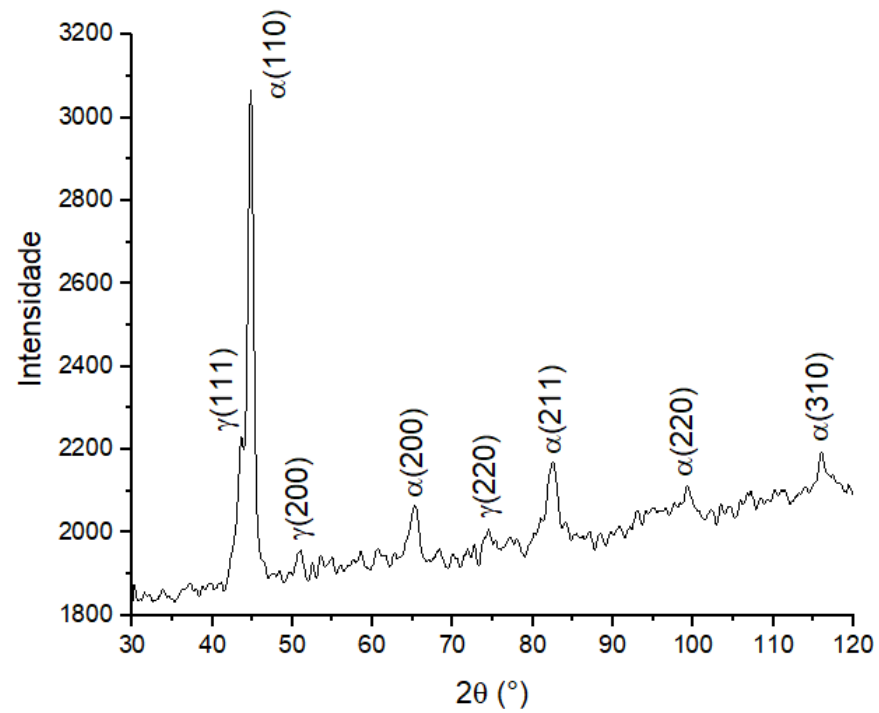

Figura 8: DRX obtida com radiação de Cu de aço SAE 1015 carbonitretado, temperado e revenido apresentando picos de austenita retida.

\section{CONCLUSÕES}

A partir dos resultados e discussões, conclui-se que:

- A camada carbonitretada apresentou-se homogênea com a presença de martensita e nitretos de elevada microdureza.

- A presença de potencial de carbono muito elevado foi favorável para formação de austenita retida e a presença de cementita em contorno de grão, na camada carbonitretada. Este fato acarretou um gradiente de microdureza e elevação gradativa da microdureza, resistência mecânica e ao desgaste na engrenagem, junto a superfície.

- A presença de austenita retida pode ocasionar uma futura transformação martensítica por deformação plástica.

- Houve um aumento significativo da microdureza superficial, contribuindo para o aumento da resistência ao desgaste e para o aumento da vida em fadiga dos componentes pela presenta de tensões compressivas da superfície (DRX).

- Os picos de DRX identificaram comportamento típico de aço baixo carbono e baixa liga carbonitretado quanto as fases detectadas, apresentando os planos de difração das estruturas CCC (ferrita e Martensita) na zona de transição e CFC (austenita) confirmando a presença de austenita retida na camada carbonitretada.

- A profundidade da camada carbonitretada encontrada foi de $0,8 \mathrm{~mm}$.

\section{BIBLIOGRAFIA}

[1] TAWEEJUN, N., KANCHANOMAI, C. "Effects of Carbon and Nitrogen on the Microstructure and Mechanical Properties of Carbonitrided Low-Carbon Steel," doi: 10.1007/s11665-015-1757-x. J. Mater. Eng. Perform., v. 24, n. 12, pp. 4853-4862, 2015.

[2] EL-HOSSARY, F.M., NEGM, N.Z., ABD EL-RAHMAN, A.M., et al. "Duplex treatment of 304 AISI stainless steel using rf plasma nitriding and carbonitriding," doi: 10.1016/j.msec.2008.09.049. Mater. Sci. Eng. C, v. 29, n. 4, pp. 1167-1173, 2009.

[3] VETTER, J., BARBEZAT, G., CRUMMENAUER, J., et al., "Surface treatment selections for automotive applications," doi: 10.1016/j.surfcoat.2005.08.011. Surf. Coatings Technol., v. 200, n. 5-6, pp. 1962-1968, 2005.

[4] BRANDOLT, C.S., GONÇALVES, F.V., SAVARIS, I.D., et al., "The influence of the tempering temperature on hydrogen embrittlement in carbonitrided modified SAE $10 \mathrm{~B} 22$ steel," doi: 10.1002/maco.201508607. Mater. Corros., v. 67, n. 5, pp. 449-462, 2016,

[5] TAWEEJUN, N., POAPONGSAKORN, P., KANCHANOMAI, C. "Microstructure and Mechanical Properties of Resistance Spot Welding Joints of Carbonitrided Low-Carbon Steels," doi: 10.1007/s11663- 
017-0927-7. Metall. Mater. Trans. B Process Metall. Mater. Process. Sci., v. 48, n. 2, pp. 1174-1187, 2017,

[6] VIEIRA, E.R., BIEHL, L.V., MEDEIROS, J.L.B., et al., "Effects of the variation of the concentration of aqueous polymer solution based on PVP in the quenching of AISI 4140 steel," doi: 10.1590/s1517707620190003.0740. Rev. Mater., v. 24, n. 3, 2019.

[7] LIU, B., WANG, B., GU, J. "Effect of ammonia addition on microstructure and wear performance of carbonitrided high carbon bearing steel AISI 52100," doi: 10.1016/j.surfcoat.2019.01.019. Surf. Coatings Technol., v. 361, pp. 112-118, Jan.2019.

[8] MEDEIROS, J.L.B., REGULY, A., STROHAECKER, R.T. “Applying Oxi-nitrocarburizing surface strengthening process to corrosion prevention in MIM 17-4 PH Stainless Steels," Espac., v. 36, n. 20, pp. $21-$ 26, 2015.

[9] ASTM International, “ASTM E3-11(2017) Preparation of Metallographic Specimens 1,” Area, vol. 03, no. July, pp. 1-17, 2001, doi: 10.1520/E0003-11R17.1.

[10] DEDAVID, B.A., GOMES, C.I., MACHADO, G. Microscopia eletrônica de varredura: Aplicações e preparação de amostras. Porto Alegre: Edipucrs, 2007.

[11] ASTM International, "E384 -17 Standard Test Method for Microindentation Hardness of Materials," doi: 10.1520/E0384-17. ASTM Int., pp. 1-40, 2017,

[12] XIONG, Z.P., KOSTRYZHEV, A.G., STANFORD, N.E., et al. "Microstructures and mechanical properties of dual phase steel produced by laboratory simulated strip casting," doi: 10.1016/j.matdes.2015.09.031. Mater. Des., v. 88, pp. 537-549, 2015.

[13] KANCHANOMAI, C., LIMTRAKARN, W. "Effect of residual stress on fatigue failure of carbonitrided low-carbon steel," doi: 10.1007/s11665-008-9212-x. J. Mater. Eng. Perform., v. 17, n. 6, pp. 879-887, 2008.

[14] KUSMANOV, S.A., KUSMANOVA, Y.V., SMIRNOV, A.A., et al., "Modification of steel surface by plasma electrolytic saturation with nitrogen and carbon," doi: 10.1016/j.matchemphys.2016.03.011. Mater. Chem. Phys., v. 175, pp. 164-171, 2016.

[15] EMADODDIN, E., AKBARZADEH, A., DANESHI, G. "Effect of intercritical annealing on retained austenite characterization in textured TRIP-assisted steel sheet," doi: 10.1016/j.matchar.2006.04.006. Mater. Charact., v. 57, n. 4-5, pp. 408-413, 2006.

[16] OLIVEIRA, R.C.L.M., BIEHL, L.V., MEDEIROS, J.L.B., et al. "Análise comparativa entre a têmpera e partição versus a têmpera e revenimento para o aço SAE 4340,” doi: 10.1590/s1517-707620190003.0788. Matéria (Rio Janeiro), v. 24, n. 3, 2019.

[17] VIEIRA, E.R., BIEHL, L.V., MEDEIROS, J.L.B., et al., "Evaluation of the characteristics of an AISI 1045 steel quenched in different concentration of polymer solutions of polyvinylpyrrolidone," Sci. Rep., vol. 11, no. 1, pp. 1-8, 2021, doi: 10.1038/s41598-020-79060-0.

\section{ORCID}

Hektor Oliveira Borges

Angelo Ores Bonamigo

Raí Lima Vieira

Jorge Luis Braz Medeiros

Luciano Volcanoglo Biehl

José de Souza https://orcid.org/0000-0002-3843-7959

https://orcid.org/0000-0001-5721-5481

https://orcid.org/0000-0002-0784-3217

https://orcid.org/0000-0003-3468-7632

https://orcid.org/0000-0002-5415-5863

https://orcid.org/0000-0001-5888-840X 